\title{
Current evidence on Multifetal pregnancy reduction and selective termination
}

\author{
Anju Bhatia ${ }^{1}$ and George Seow-Heong Yeo ${ }^{2}$ \\ ${ }^{1} \mathrm{KK}$ Women's and Children's Hospital \\ ${ }^{2}$ KK Womens and Childrens Hospital
}

April 28, 2020

\begin{abstract}
Multifetal gestation, which accounts for approximately $3 \%$ of births, is a high-risk pregnancy being associated with adverse obstetric and perinatal complications with its consequences including neurodevelopmental handicaps. Multifetal pregnancy reduction aims to reduce the risk of miscarriage and extreme preterm birth at an acceptable procedure-related loss rate by terminating one or more fetuses. Selective termination for discordant anomalies in monochorionic pregnancies has advanced in recent years with the development of various vascular-occlusive techniques. Obstetricians should be knowledgeable about the medical risks of multifetal pregnancy, the potential benefits of these interventions and complex ethical issues inherent in the decisions.
\end{abstract}

\section{Introduction}

Multifetal pregnancy is one of the greatest perinatal challenges. Changing demographics and fertility practice has resulted in the global epidemic of multifetal pregnancies which has substantially inflated the perinatal disease burden for clinicians, parents as well as society. ${ }^{1,2}$ Bergh $^{3}$ attributed a third of the increase to in-vitro fertilization(IVF), a third to ovulation induction and rest to advanced maternal age. Lawlor and Nelson et $\mathrm{al}^{4}$ demonstrated that the success rate for live-borns was higher by about $7 \%$ by transferring 2 embryos in 1 cycle rather than 1 each for 2 cycles. Thus, where aggressive embryo transfers seem justifiable, mounting multiple pregnancies are to be dealt with.Evans ${ }^{5}$ reviewed trends over past 3 decades and reported that twin births have surged high and stayed relatively constant, but the curves for triplets and above show a curvilinear pattern, an incidence which skyrocketed initially, has returned to almost baseline due to Human Fertilization and Embryology Authority Code of Practice (2001), which restricted the number of embryos transferred at IVF/ICSI cycle to two.

The incidence of complications in multifetal pregnancy directly correlates with the number of fetuses. Besides the higher risk of miscarriages, infants born are at increased risk of prematurity, cerebral palsy, chronic lung disease, developmental delay, behavioral difficulties and death. ${ }^{6-8}$ Advancements in neonatal care may have reduced associated mortality though morbidity has remained unabated. Pharoah and Cooke reported cerebral palsy rates per 1,000 $1^{\text {st }}$ year survivor at 2.3 for singletons, 12.6 for twins, and 44.8 for triplets. ${ }^{9,10}$ Complications secondary to unique placental vascular connections further escalates risks for monochorionic gestations. Maternal risks include hypertension, preeclampsia, gestational diabetes, postpartum hemorrhage. ${ }^{11}$

Sub-fertile couples may consider multiple pregnancies as a desirable outcome after trying many years but associated high rate of maternal and perinatal complications has compelled obstetricians to consider prevention options. Primary prevention has been achieved to an extent with sonographic monitoring of ovulation induction with cancellation if excessive ovulation is predicted, and the restrictions on embryo transfer. However, 
once twins or higher-order multiple pregnancy has occurred, the option of multifetal pregnancy reduction or selective termination (ST) needs to be considered as a secondary prevention measure. Fetal reduction not only improves medical outcomes but also significantly reduces the economic and psychological impact of caring for multiple new-borns.

\section{Multifetal pregnancy reduction (MFPR)}

MFPR refers to an intervention in which the number of fetuses in a multifetal gestation is reduced by terminating one or more fetuses.

The first reports of MFPR came from France by Dumez and Oury in 1986 explaining first-trimester selective abortion for pregnancies involving more than 3 fetuses, or an abnormal cotwin. ${ }^{12}$ Evans et al ${ }^{13}$ reported their MFPR experience and argued it to be ethically justified because it met the criterion of least harm and more potential good. Berkowitz ${ }^{14}$ and later Wapner ${ }^{15}$ described a surgical approach to improve the outcomes. Methods have included predominately transthoracic injection of Potassium Chloride ( $\mathrm{KCl})$, but also transcervical embryo aspiration, mechanical disruption of the fetus, air embolization, and electrocautery.

American College of Obstetricians and Gynaecologists (ACOG) Committee Opinion ${ }^{16}$ on MFPR recommends that obstetricians should be knowledgeable about medical risks of multifetal pregnancy, potential benefits of MFPR and complex ethical issues inherent in the decision for reduction. Nondirective patient counseling should be offered to all women with multifetal pregnancies by a multidisciplinary team including maternalfetal medicine specialists, neonatologists, child development specialists, mental health professionals, etc. and informed consent taken. Patient should be referred in time and without judgement to fetal therapy center. Obstetrician should respect patients' autonomy and that "only the patient can weigh the relative importance of the medical, ethical, religious, and socioeconomic factors and determine the best course of action for her unique situation."

The consensus views arising from the 50th RCOG Study Group recommend that selective reduction should be discussed in all higher-order pregnancies. ${ }^{17}$ An International Federation of Gynaecology and Obstetrics(FIGO) Committee Report ${ }^{18}$ stated "multiple pregnancy of an order of magnitude higher than twins involves great danger for the woman's health and also for her fetuses, which are likely to be delivered prematurely with a high risk of either dying or suffering damage" and "where such pregnancies arise, it may be considered ethically preferable to reduce the number of fetuses rather than to do nothing".

MFPR is performed between 11 and 14 weeks as the risk of spontaneous reduction has by then passed ${ }^{19}$ and fetus are sufficiently large to allow for the detection of gross structural anomalies and markers of aneuploidy including thick nuchal translucency or absent nasal bone to guide the selection of the fetus(es) for reduction. Pregnancies conceived by IVF and ICSI are known to be associated with a higher incidence of aneuploidy as well as structural anomalies, some centers now offer routinely chorionic villous sampling (CVS) before the MFPR. Evans ${ }^{20}$ reported about $85 \%$ of their patients followed the protocol of CVS followed by MFPR the next day. In pregnancies with sonographically normal fetuses, $3.1 \%$ of women had a fetus with an abnormal karyotype. FISH detected $90 \%$ of aneuploidy while the remaining $10 \%$, virtually were confined placental mosaicisms for other chromosomes. ${ }^{21}$

There are no absolute contraindications to MFPR, however, cases as a mother with HIV or active hepatitis, extreme obesity with poor visualization of the fetus warrant special consideration.

The fetus(es) selected for MFPR is generally the one that is most accessible to needle insertion and furthest away from the cervix. However, if a fetus has a structural anomaly, markers of aneuploidy or lagging crownrump length or a substantially smaller sac then that fetus is preferentially chosen for reduction so as to leave behind apparently healthy fetuses. Most commonly, the procedure is performed using a transabdominal percutaneous technique under ultrasound guidance. A transcervical or a transvaginal approach can be used if performed before10 weeks or transabdominal route is not technically feasible. However, recent studies have confirmed that these routes have been associated with higher postprocedural pregnancy loss rates. ${ }^{22}$

MFPR is performed most commonly by giving an intracardiac injection of $\mathrm{KCl}(1.5 \mathrm{~g}$ in $10 \mathrm{~mL})$ into the 
targeted fetus using a 20 gauge needle resulting in fetal asystole. Additional fetuses can be reduced by the same puncture if next desired fetus is accessible or else a separate second puncture may be required to complete the procedure. An ultrasound examination is performed one hour after the procedure to confirm asystole in the reduced fetus(es) and cardiac activity in the nonreduced fetus(es).

A finishing number of two has become standard practice, as the perinatal outcome of twin pregnancies is considered acceptable and it still leaves an option of ST if discordant fetal abnormalities are detected at mid-trimester scan later.

Data from observational studies ${ }^{23,24}$ show consistently that perinatal and obstetric outcomes particularly preterm birth (PTB) rates, improve significantly after MFPR. International Registry analysis of 3513 patients undergoing MFPR, in 11 centers showed overall loss rates were correlated strongly with starting and finishing numbers. Stone et $\mathrm{al}^{24}$ reported outcomes of 1000 consecutive cases of MFPR at Mount Sinai Medical Centre (1999-2006). Post-procedure pregnancy loss rate was $4.7 \%$ and it correlated with the starting number when reduced to twins (> 5: $12.1 \%$ loss; 4: $5.8 \%$ loss; $3: 4.5 \%$ loss), but that the rate of PTB did not differ with starting number. The lowest loss rate occurred in the patients reducing from twins to a singleton (2.1\%), likely it is basically the background spontaneous loss rate of twin pregnancies. ${ }^{25,26}$ Reduction to a singleton was also associated with higher birth-weights and lower rates of preterm deliveries.

Evidence of improved perinatal and obstetric outcomes after reduction of $>4$ fetuses is undisputed, convincing data has emerged for triplets as well in various meta-analysis.

A 2017 meta-analysis ${ }^{27}$ including 24 studies (1999-2015) compared triplets reduced to twins with expectantly managed triplets. The risk of pregnancy loss before 24 weeks was similar in both groups $(7.4$ versus $8.1 \%$, OR $0.87,95 \%$ CI $0.52-1.42)$. Reduction to twins was associated with a $60 \%$ reduction in early PTB ( $<32$ weeks: OR $0.30,95 \%$ CI $0.22-0.41 ;<28$ weeks: OR $0.40,95 \%$ CI $0.22-0.71)$ which resulted in a significant reduction in neonatal mortality. Other adverse pregnancy outcomes as gestational diabetes reduced by nearly $70 \%$ and hypertensive disorders by $60 \%$. Mothers of reduced triplets had a lower rate of Caesarean deliveries $(61.4 \%)$ compared with the non-reduced triplets' $(90.5 \%)$ cohort. Nonreduced triplets delivered significantly earlier, with a consequent higher prevalence of major morbidities. ${ }^{28}$ Twins from reduced triplets didn't follow the true growth curves of spontaneous twins, and instead, retain their suboptimal growth potential as triplets, secondary to early placental processes or retained fetoplacental material after MFPR.

Papageorghiou et $\mathrm{al}^{29}$ calculated that seven (95\%CI 5-9) reductions needed to be performed to prevent one early PTB, while the number of reductions that would cause one miscarriage was $26(95 \% \mathrm{CI} 14-193)$ in their systematic review.

Another meta-analysis ${ }^{30}$ separately assessed outcomes of trichorionic triamniotic triplet pregnancies (TCTA, $\mathrm{n}=501)$ and dichorionic triamniotic triplet pregnancies (DCTA, $n=200$ ) versus those electively reduced to twins (TCTA $n=666$;DCTA $n=49$ ). In TCTA gestations, MFPR was associated with a substantial reduction in PTB $<34$ weeks (17.3 versus $50.2 \%$ ), without a substantial increase in the rate of miscarriage (8.1 versus $7.4 \%$ ). In DCTA pregnancies, only a modest reduction of PTB ( $51.9 \%$ to $46.2 \%$ ) was observed with selective reduction of the triplet with the separate placenta while miscarriage rate increased from 8.5 to $13.3 \%$. The numbers were small to draw any confident conclusion.

Morlando ${ }^{31}$ presented a systemic review of MFPR outcomes. Out of 331 DCTA triplets, the miscarriage and PTB rates were $8.9 \%$ and $33.3 \%$ respectively with expectant management. The miscarriage rate was $14.5 \%$ with a reduction of MC pair, $8.8 \%$ with a reduction of one fetus of the monochorionic pair, and $23.5 \%$ with a reduction of the fetus with a separate placenta. Severe PTB rates were $5.5 \%, 11.8 \%$, and $17.6 \%$ respectively. The highest rate of fetal loss was observed where monochorionic pair was left behind reflecting losses secondary to complications of monochorionicity. Hence, they concluded expectant management in DCTA triplets, is a reasonable choice when a liveborn infant is a top priority irrespective of the possible presence of handicap. The benefit of fetal reduction is a decrease in the rate of severe PTB thereby minimize risks of long-term sequelae. 
Presently enough evidence exists that even when chorionicity is taken into consideration, reducing triplets to twins does reduce the rate of PTB with probably no significant increase in the miscarriage rate.

A recent retrospective review ${ }^{32}$ of large contemporary cohort comparing pregnancy outcomes of DCDA twin pregnancies reduced to singletons $(\mathrm{n}=250)$ with ongoing (nonreduced) twin pregnancies $(\mathrm{n}=605)$ showed in univariate logistic regression analysis, MFPR was associated with higher mean gestational age at delivery (39 versus 36.7 weeks), reduction in PTB $<37$ weeks (18 versus $54 \%$ ) and $<34$ weeks $(7$ versus $17 \%$ ) as well as reduction in birth weight $<5^{\text {th }}$ percentile $(9$ versus $22 \%$ ). Other adverse outcomes including preeclampsia (4 versus 17\%), PPROM (6 versus 19\%), gestational diabetes (5 versus $9 \%$ ) also showed improvement with MFPR. No significant increase in unintended pregnancy loss $<24$ weeks (2.4 versus $2.3 \%$ ) was reported.

\section{SELECTIVE TERMINATION}

The incidence of fetal malformations is known to be higher in multifetal pregnancies compared to singletons. ${ }^{33}$ In dizygotic twins, the risk of malformation is slightly more than twice the risk per pregnancy (independent probabilities per fetus). In monozygotic twins, Mendelian and chromosomal abnormalities are comparable to singletons, while the definite higher risk of structural malformations exists. In nearly $85 \%$ of cases irrespective of chorionicity, the malformation is confined to one fetus. After the prenatal diagnosis, a couple would have three options: expectant management, terminate both normal and abnormal fetuses, or ST of the abnormal fetus.

Aberg $^{34}$ reported the first successful selective birth from a twin pregnancy discordant for Hurler syndrome in 1978. Kerenyi and Chitkara ${ }^{35}$ followed with a report of ST for twins discordant for Down syndrome in 1981. Throughout the 1980s a number of small series of second-trimester selective terminations appeared in the literature, most of which showed very high loss rates and morbidity. ${ }^{36-38}$ The high loss rates represented were ultimately determined to be principally in monochorionic pregnancies.

Selective termination refers to an intervention in which one or more specific fetuses with a known or suspected fetal chromosomal, structural, or genetic abnormality of a multifetal gestation are terminated.

ST was initially offered as an option to mothers with fetuses with major nonlethal anomalies that could otherwise lead to the live birth and long-term survival of a severely impaired child. ST is now also offered for fetuses with lethal anomalies because some mothers find it emotionally challenging to carry on baby with a gross fetal anomaly like anencephaly that will die before or shortly after birth. Secondly associated antenatal issues as polyhydramnios or hydrops may increase the risk of PTB.

The main variable in selecting the technique of ST is the chorionicity. In dichorionic (DC) twins, intracardiac $\mathrm{KCl}$ in the affected fetus is safe for the normal co-twin. In monochorionic(MC) pregnancies, the risk of passage of $\mathrm{KCl}$ into the circulation of the normal co-twin through placental anastomosis precludes this technique. Hence, the accurate determination of chorionicity between 9-13 weeks is crucial before performing ST.

\section{Dichorionic multiple gestations}

Selective termination can be easily carried out in dichorionic gestations by the transabdominal percutaneous procedure. ${ }^{39}$ Under ultrasound guidance, the intracardiac injection of $\mathrm{KCl}$ is given to the targeted fetus using a 20 or 22 -gauge needle until asystole is confirmed.

Evans ${ }^{40}$ published the largest multicenter series on outcomes of ST in dizygotic pregnancies for structural or chromosomal anomalies. They reported loss rates with gestation of procedure with rates of $5.4 \%$ at $9-12$ weeks, $8.7 \%$ at $13-18$ weeks and $9.1 \%$ at $>25$ weeks, although these did not reach significance. The mean gestation at delivery was 35.7 weeks with a liveborn infant in $>90 \%$ of cases. They concluded ST, in the most experienced hands, can be technically performed in all 3 trimesters (if legal) with good outcomes in $>90 \%$ of cases.

This report was followed by several single-center series ${ }^{41-43}$ which confirmed ST in DC pregnancy with discordant anomaly to be a safe, feasible and effective option. With experience, the procedure-loss rate is reported as $4 \%$ and mean gestational age at delivery as 38 weeks. 


\section{Monochorionic multiple gestations}

$\mathrm{MC}$ twins are at substantially increased risk of adverse outcomes compared to DC twins, ${ }^{44}$ secondary to typical angioarchitecture of a monochorionic placenta. ${ }^{45}$ Vascular anastomoses maintain a dynamic bidirectional flow between the co-twins. Unique complications such as twin-to-twin transfusion syndrome, twin reversed arterial perfusion sequence, selective intrauterine growth restriction or discordant malformations are encountered in monochorionic gestations due to altered vascular connections. When one of the twins suffers major structural anomaly or shows doppler patterns suggestive of impending demise, ST of the specific fetus is the only therapeutic option available to optimize the survival of co-twin. In case of intrauterine demise of one fetus, co-twin may acutely exsanguinate across vascular anastomoses and die or suffer neurological damage in $30-50 \%$ cases. $^{46}$

ST using various vaso-occlusive techniques that completely occlude flow through the targeted fetus's umbilical cord have been developed. ${ }^{47}$ Initial attempts were made with cord ligation or cord embolization with thrombogenic coils or sclerosants. These were often complicated by method failure and high pregnancy loss-rates. Presently techniques used are namely bipolar cord coagulation (BCC), laser cord coagulation, radiofrequency ablation (RFA), microwave ablation and High Intensity Focussed Ultrasound to achieve selective termination. The main complications associated with these interventions are preterm rupture of membranes (PPROM), hemorrhage and PTB.

Bipolar cord coagulation is the standard procedure for ST in monochorionic pregnancies. Under ultrasound guidance, a $3 \mathrm{~mm}$ port is inserted into the sac of the affected fetus and a bipolar coagulation forceps is passed and used to grasp the cord of the affected fetus which is coagulated using bipolar energy between 20 and $50 \mathrm{~W}$. This technique simultaneously obliterates the umbilical arteries and vein, causing immediate cessation of flow and thus preventing any interfetal hemorrhage. Overall survival rates for co-twin are between $76 \%$ and $88 \%$ with low risk of neurological damage $(2.6 \%){ }^{48-50}$

Radiofrequency ablation uses a $17 \mathrm{G}$ radiofrequency electrode to generate high-frequency sinusoidal current $(400-500 \mathrm{kHz})$ which induces local tissue ionic agitation resulting in frictional heat to cause thermal coagulation. Under ultrasound guidance, using local anesthesia RFA needle is inserted into the fetal abdomen at the level of cord insertion, guided by color Doppler mapping. Radiofrequency energy is applied until there is 'roll-off'. Tissue necrosis results in increased tissue impedance that prohibits the passage of electrical current, hence there is a drop-in power output indicated as roll-off. Coagulative damage is usually restricted to $2 \mathrm{~cm}$ area around the needle tip. Commonly used devices include LeVeen RFA probe (Boston Scientific, Natick, MA), Starburst SDE_ radiofrequency needle (Angiodynamics, Inc., Queensbury, NY, USA) and Covidien Cool-tip RF ablation system (Medtronics) Each function slightly differently, but the net effect achieved is the same. Occlusion of blood flow occurs slowly only once adequate power has been applied to achieve tissue desiccation. This may result in an increased risk of thermal damage to membranes and co-twin demise due to acute exsanguination.

Fetoscopically guided Nd:YAG laser coagulation of the cord or the vessels at the route of the cord has been used for ST in MC gestations. A 400-600-micron laser fiber positioned under ultrasound guidance in the fetoscope and is advanced a few millimeters beyond the tip of the needle into the target tissue. Power is applied in short bursts until the blood flow stops. Coagulation occurs slowly only once adequate thermal energy is applied often requiring multiple applications of laser. Slower occlusion again increases the risks for co-twin.

Newer techniques employed are microwave ablation or High frequency focused ultrasound (HIFU). A thin microwave antenna is placed directly into the fetus using ultrasound guidance. This antenna emits an electromagnetic wave through its exposed, non-insulated tip which agitates water molecules in the surrounding tissue, producing friction and heat, thus, inducing cellular death via coagulation necrosis. ${ }^{51}$ The antennae are internally cooled with either room-temperature fluid or carbon dioxide to reduce conductive heating and to prevent possible skin damage.

Modality of HIFU has been used to manage TRAP sequence cases. ${ }^{52}$ This technique involves the transmission 
of a beam of ultrasound through solid tissue which is focused within the fetus, enabling thermal coagulation necrosis. The chief advantage lies in being truly non-invasive. However only a few reports are published and in one of them the co-twin was delivered with pseudoarthosis ${ }^{52}$, and the role of ultrasound beam is not clear. The procedure required several attempts to achieve complete occlusion, thus leaving the potential for transfusion-related complications.

Rossi et al $^{50}$ presented a systematic review of 345 complicated MC twins who underwent ST. Overall survival rate is almost similar for BCC $(82 \%)$ or RFA $(86 \%)$. Nearly $22 \%$ had PPROM and in one-sixth of cohort, co-twin demise was reported. Roman et al ${ }^{49}$ also showed no clear superiority of any one technique in their comparative series.

Bebbington $^{53}$ reported that $\mathrm{BCC}$ was associated with a higher rate of overall survival $(85.2 \% \mathrm{v} 70.7 \%)$ compared to those who had RFA for ST. The difference was attributed to an increased survival rate of the co-twin below 28 weeks (BCC 31.6\% Vs RFA 10.5 \%) probably as there was a complete cessation of blood flow in BCC cases whereas, in RFA, there is slow cessation.

O'Donoghue ${ }^{54}$ reported outcomes for the use of interstitial laser in ST of thirty MC pregnancies. Overall perinatal survival was reported in $68 \%$ cases. Two of 26 neonates $(8 \%)$ were diagnosed with aplasia cutis congenita. They postulated that the procedure-related losses may be related to difficulty in maintaining the correct position of the laser fibre during repeated laser applications. However, Jennifer ${ }^{55}$ demonstrated laser cord occlusion is a reasonable surgical modality for ST in their retrospective review with high PPROM $(32.6 \%)$ and co-twin survival rates $(95.3 \%)$.

Meng et $\mathrm{al}^{56}$ described their preliminary novel experience in the application of microwave ablation for ST and reported it to be safe and effective. None of the surviving co-twins had evidence of thermal injury or neurological abnormalities and the overall survival rate was $73.3 \%(\mathrm{n}=33 / 45)$.

All techniques for ST are associated with some risk of co-twin demise and complications such as miscarriage, PPROM, preterm birth, etc. (Table 1). Each of ST technique has its own merits, no single perfect technique exists to manage complicated MC gestations. No randomized control studies are available to assess the superiority of one method over others, the evidence is though available through various systematic reviews and metanalyses. The balance between benefits and risks with these procedures is not always completely clear and the decision is usually based on technical considerations, local experience, available instrumentation and above all parental wishes.

Conclusion: Management of multifetal pregnancies is challenging due to the associated high rates of complications. MFPR aims to increase the likelihood of a successful pregnancy and the best current means of evading undesired sequelae of extreme prematurity associated with high-order multiples. Selective termination for anomalies in multifetal pregnancies has significantly advanced with acceptable low procedure-related loss rates. Couples should be provided balanced counseling about benefits and risks associated with these fetal interventions so that they can make informed choices for their pregnancy.

Conflict of Interest: All authors declare that there are no conflicts of interest.

Contribution to authorship: AB did the literature search and wrote the article. YSH gave expert opinion and contributed to the conclusion. All authors approved the final version.

Funding Information: No funding was obtained for this study.

\section{References}

1. Office for National Statistics. Birth Statistics 2008. London: Office for National Statistics; 2010. FM1 No. 37: 30-33.

2. Hall J. Twinning. Lancet 2003;362(9385):735-43.

3. Bergh T, Ericson A, Hillensjo T, Nygren KG, Wennerholm UB. Deliveries and children born after in-vitro fertilisation in Sweden 1982-95: a retrospective cohort study. Lancet 1999;354(9190):1579-85. 
4. Lawlor DA, Nelson SM: Effect of age on decisions about the number of embryos to transfer in assisted conception: a prospective study. Lancet 2012; 379: 521-527.

5. Evans M, Andriole S, Britt DW. Featl reduction: 25years experience. Fetal Diagn Ther 2014; 35:69-82.

6. Multiple gestation pregnancy. The ESHRE Capri Workshop Group. Hum Reprod 2000; 15:1856-64.

7. Multifetal gestations: twin, triplet and higher-order multifetal pregnancies. Practice Bulletin No. 169. American College of Obstetricians and Gynacologists. Obstet Gynecol2016;128: e131-46.

8. Perinatal risks associated with assisted reproductive technology. Committee Opinion No. 671. American College of Obstetricians and Gynacologists. Obstet Gynecol2016;128: e61-8.

9. Pharoah PO, Cooke T: Cerebral palsy and multiple births. Arch Dis Child Fetal Neonatal Ed 1996; 75: F174-F177.

10. Dimitiiou G, Pharoah PO, Nicolaides KH, et al: Cerebral palsy in triplet pregnancies with and without iatrogenic reduction. Eur J Pediatr 2004; 163: 449-45.

11. Luke B, Brown MB. Contemporary risks of maternal morbidity and adverse outcomes with increasing maternal age and plurality. Fertil Steril2007; 88:283-93.

12. Dumez Y, Oury JF: Method for first trimester selective abortion in multiple pregnancy. Contrib Gynecol Obstet 1986; 15: 50-53.

13. Evans MI, Fletcher JC, Zador IE, Newton BW, Struyk CK, Quigg MH: Selective first trimester termination in octuplet and quadruplet pregnancies: clinical and ethical issues. Obstet Gynecol 1988; 71: 289-296.

14. Berkowitz RL, Lynch L, Chitkara U, et al: Selective reduction of multiple pregnancies in the first trimester. N Engl J Med 1988; 318: 1043.

15. Wapner RJ, Davis GH, Johnson A: Selective reduction of multifetal pregnancies. Lancet 1990; 335: 90-93.

16. Committee on Ethics. Committee Opinion No. 719: Multifetal Pregnancy Reduction. Obstet Gynecol 2017; 130: e158.

17. Kilby MD, Baker PN, Critchley HO, Field DJ, editors. Consensus views arising from the $50^{\text {th }}$ Study Group: Multiple pregnancy. In: Multiple pregnancy. London: RCOG Press; 2006.p.283 - 6.

18. FIGO Committee for the Ethical aspects of Human Reproduction and Women's Health. Ethical recommendations on multiple pregnancy and multifetal reduction. Int J Gynaecol Obstet 2006; 92:331.

19. Landy HJ, Keith LG. The vanishing twin: a review. Hum Reprod Update 1998; 4:177-83.

20. Evans MI, Britt DW. Fetal reduction. Semin Perinatol 2005; 29:321-9.

21. Rosner M, Pergament E, Andriole S, Gebb J, Dar P, Evans MI: Detection of genetic abnormalities using CVS and FISH prior to fetal reduction in sonographically normal appearing fetuses. Prenat Diagn 2013; 33: 940-944.

22. M.S.Kim, D.H.Choi, J.E.Shin. Procedural and obstetric outcomes after embryo reduction vs fetal reduction in multifetal pregnancy Ultrasound Obstet Gynecol 2019; 53: 214-218.

23. Evans MI, Berkowitz RL, Wapner RJ, Kypros H Nicolaides. Improvement in outcomes of multifetal pregnancy reduction with increased experience. Am J Obstet Gynaecol 2001; 184:97-103.

24. Stone J, Ferrara L, Kamrath J et al. Contemporary outcomes with the latest 1000 cases of multifetal pregnancy reduction (MPR). Am J Obstet Gynaecol 2008; 199:406. e1.

25. Ward Platt MP, Glinianaia SV, Rankin J,Wright C, Renwick M. The North of England Multiple Pregnancy Register: five-year results of data collection. Twin Res Hum Genet 2006; 9(6):913-8.

26. Stone J, Eddleman K. Multifetal pregnancy reduction. Curr Opin Obstet Gynecol 2000;12 491-6.

27. Zipori Y, Haas J, Berger H, Brazilay E. Multifetal pregnancy reduction of triplets to twins compared with non-reduced triplets: a meta-analysis. Reprod Biomed Online 2017; 35:296.

28. Horbar J.D., Carpenter J.H., Badger G.J., Kenny M.J., Soll R.F., Morrow K.A., Buzas J.S. Mortality and neo-natal morbidity among infants 501 to 1500 grams from 2000 to 2009. Pediatrics 2012;129, 1019-1026.

29. Papageorghiou AT, Avgidou K, Bakoulas V, Sebire NJ, Nicolaides KH. Risks of miscarriage and early preterm birth in trichorionic triplet pregnancies with embryo reduction versus expectant management: new data and systematic review. Hum Reprod 2006; 21:1912-7. 
30. Anthoulakis C, Dagklis T, Mamopoulos A, Athanasiadis A.Risks of miscarriage or preterm delivery in trichorionic and dichorionic triplet pregnancies with embryo reduction versus expectant management: a systematic review and meta-analysis. Hum Reprod 2017; 32:1351.

31. Morlando M, Ferrara L et al Dichorionic triplet pregnancies: risk of miscarriage and severe preterm delivery with fetal reduction versus expectant management. Outcomes of a cohort study and systematic review BJOG April 2015,1053-60.

32. Vieira LA, Warren L, Pan S, et al. Comparing pregnancy outcomes and loss rates in elective twin pregnancy reduction with ongoing twin gestations in a large contemporary cohort. Am J Obstet Gynaecol 2019; 221(3): 253e1.

33. Luke B. Monozygotic twinning as a congenital defect and congenital defects in monozygotic twins. Fetal Diagn Ther 1990; 5:61-9.

34. Aberg A, Metelman F, Cantz M, Gehler J. Cardiac puncture of fetus with Hurler's disease avoiding abortion of unaffected cotwin. Lancet 1978; 2:990-9.

35. Kerenyi T, Chitkara U. Selective birth in twin pregnancy with discordancy for Down's syndrome. N Engl J Med 1978; 304:1525-7.

36. Rodeck C. Fetoscopy in the management of twin pregnancies discordant for a severe abnormality. Acta Genet Med Gemellol 1984; 33:57-60.

37. Rodeck C, Mibashan R, Abramowicz J, Campbell S. Selective feticide of the affected twin by fetoscopic air embolization. Prenat Diagn 1982; 2:189-94.

38. Golbus MS, Cunningham N, Goldberg JD, Anderson R, Filly R, Callen P. Selective termination of multiple gestations. Am J Med Genet 1988; 31:339-48.

39. Westendorp AK, Miny P, Holzgreve W, DeWilde R, Aydinli K. Selective fetocide by direct injection of isotonic potassium chloride. Arch Gynecol Obstet 1988; 244:59-62.

40. Evans MI, Goldberg JD, Horenstein J, et al. Selective termination for structural, chromosomal, and Mendelian anomalies: international experience. Am J Obstet Gynecol 1999; 181:893-7.

41. Eddleman KA, Stone JI, Lynch L, Berkowitz RL. Selective termination of anomalous fetuses in multifetal pregnancies: two hundred cases at a single centre. Am J Obstet Gynecol 2002; 187:1168-1172.

42. Rustico MA, Baietti MG, Coviello D, Orlandi E, Nicolini U. Managing twins discordant for fetal anomaly. Prenat Diagn 2005; 25:766-71.

43. Alvarado EA, Pacheco RP, Alderete FG, Luís JA, de la Cruz AA, Quintana LO. Selective termination in dichorionic twins discordant for congenital defect. Eur J Obstet Gynecol Reprod Biol. 2012;161:8-11.

44. Sebire, N. J., Snijders, R. J., Hughes, K., Sepulveda, W., \& Nicolaides, K. H. The hidden mortality of monochorionic twin pregnancies. British Journal of Obstetrics and Gynaecology,1997;104, 1203-1207.

45. Lewi, L., Jani, J., Blickstein, I., Huber, A., Gucciardo, L., Van Mieghem, T., . . . Deprest, J. The outcome of monochorionic diamniotic twin gestations in the era of invasive fetal therapy: A prospective cohort study. Am J Obstet Gynecol 2008;199, (5), 514.e1-8.

46. Ong SS, Zamora J, Khan KS, et al. Prognosis for the co-twin following single-twin death: a systematic review. BJOG 2006; 113:992-8.

47. Wimalasundera RC. Selective reduction and termination of multiple pregnancies. Semin Fetal Neonatal Med 2010; 15:327-35.

48. Nicolini U, Poblete A, Boschetto C, Bonati F, Roberts A. Complicated monochorionic twin pregnancies: experience with bipolar cord coagulation. Am J Obstet Gynecol 2001; 185:703-7.

49. Roman A, Papanna R, Johnson A, et al. Selective reduction in complicated monochorionic pregnancies: radiofrequency ablation vs. bipolar cord coagulation. Ultrasound Obstet Gynecol 2010; 36:37-41.

50. Rossi AC, D'Addario V. Umbilical cord occlusion for selective feticide in complicated monochorionic twins: a systematic review of literature. Am J Obstet Gynecol 2009; 200:123-9.

51. Simon CJ, Dupuy DE, Mayo-Smith WW. Microwave ablation: principles and applications. Radiographics 2005;25: S69-83.

52. Okai T, Ichizuka K, Hasegawa J, et al. First successful case of non-invasive in utero treatment of TRAP sequence by high intensity focused ultrasound. Ultrasound Obstet Gynecol 2013; 42:112-4.

53. Bebbington MW, Danzer E, Moldenhauer J, et al. Radiofrequency ablation vs bipolar cord coagula- 
tion in the management of complicated monochorionic pregnancies. Ultrasound Obstet Gynecol 2012; 40:319-24.

54. O’Donoghue K, Barigye O, Pasquini L, Chappell L, Wimalasundera RC, Fisk NM. Interstitial laser therapy for fetal reduction in monochorionic multiple pregnancy: loss rate and association with aplasia cutis congenita. Prenat Diagn 2008; 28:535-43.

55. King J R, Charlotte L Conturie,Ramen H Chmait, Umbilical cord occlusion via laser coagulation in monochorionic multifetal gestations before and after 20 weeks of gestation.Fetal Diagn Ther 2017;42:916.

56. Xinlu Meng, Pengbo Yuan, Lijun Gong, Xueju Wang, Tianchen Wu Yuan Wei, Yangyu Zhao, forty-five consecutive cases of complicated monochorionic multiple pregnancy treated with microwave ablation: A single-center experience. Prenatal Diagnosis. 2019; 39:293-298.

\section{Hosted file}

Table 1 .docx available at https://authorea.com/users/313823/articles/444243-current-evidenceon-multifetal-pregnancy-reduction-and-selective-termination 